\title{
A Rare Case of Evans Syndrome in a Patient With Ulcerative Colitis
}

\author{
Kok Hoe Chan ${ }^{\mathrm{a}, \mathrm{e}}$, Su Lin Lim ${ }^{\mathrm{a}}$, Gowthami Are ${ }^{\mathrm{a}, \mathrm{b}}$, Theodore DaCosta, Jra, b, c, \\ Hamid S Shaaban a, b, d
}

\begin{abstract}
Evans syndrome (ES) is an autoimmune condition that presents with two or more cytopenias, which includes simultaneous or sequential development of warm autoimmune hemolytic anemia (AIHA) and immune thrombocytopenic purpura (ITP). The association of ES with ulcerative colitis (UC) was only reported once in the literature. Herein, we present a case of a 66-year-old male patient with a history of UC, who was diagnosed with ES secondary to UC, for which he was treated with steroids. Recognizing this rare association is important as prompt treatment with intravenous immunoglobulin and steroids will improve the prognosis and reduce the risk of complications.
\end{abstract}

Keywords: Evans syndrome; Ulcerative colitis; Autoimmune hemolytic anemia; Immune thrombocytopenic purpura

\section{Introduction}

Evans syndrome (ES) is an autoimmune condition that presents with two or more cytopenias, which includes simultaneous or sequential development of warm autoimmune hemolytic anemia (AIHA) and immune thrombocytopenic purpura (ITP) with or without immune neutropenia [1]. Although the exact mechanism is not fully understood, some studies have linked it to a decreased in cluster of differentiation 4 (CD4)/CD8 ratio [2]. ES can be primary (idiopathic) or secondary to other

Manuscript submitted July 22, 2020, accepted August 4, 2020

Published online August 14, 2020

aDepartment of Medical Education, Saint Michael's Medical Center, New York Medical College, Newark, NJ 07101, USA

bDepartment of Internal Medicine, Saint Michael's Medical Center, New York Medical College, Newark, NJ 07101, USA

'Department of Gastroenterology, Saint Michael's Medical Center, New York Medical College, Newark, NJ 07101, USA

dDepartment of Hematology/Oncology, Saint Michael's Medical Center, New York Medical College, Newark, NJ 07101, USA

eCorresponding Author: Kok Hoe Chan, Department of Medical Education, Saint Michael's Medical Center, Newark, NJ 07101, USA.

Email: Kokhoedelcos.chan@gmail.com

doi: https://doi.org/10.14740/gr1316 disorders. Although secondary ES has higher mortality rates, it responds better to treatment [3, 4]. A few secondary causes were mentioned in literature, which includes systemic lupus erythematosus (SLE), common variable immunodeficiency (CVID), autoimmune lymphoproliferative syndrome (ALPS), viral infections (human immunodeficiency viruses (HIV) and hepatitis $\mathrm{C}$ ) and hematological malignancies (chronic lymphocytic leukemia (CLL)). However, to our knowledge, the association of ES with either ulcerative colitis (UC) or prostate cancer was reported only once in the literature [5].

Herein, we present a case of a 66-year-old male patient with a history of UC, who presented to the emergency department with fatigue, shortness of breath and weakness. His blood works showed anemia, elevated white blood cell count (WBC), thrombocytopenia and indirect hyperbilirubinemia, he had low partial thromboplastin time (PTT) but normal prothrombin time/international normalized ratio (PT/INR), significantly elevated reticulocyte count and lactate dehydrogenase (LDH) with low haptoglobin. He had a positive direct antiglobulin test poly Coombs' test, warm autoantibody and immunoglobulin $\mathrm{G}$ (IgG) with negative $\mathrm{C} 3$. The patient was diagnosed with ES secondary to UC, for which he was treated with intravenous Ig (IVIG) and was sent home on steroids.

\section{Case Report}

A 66-year-old male with a past medical history significant for UC, diagnosed in 1999 on mesalamine, history of prostate cancer in remission status post radical prostatectomy for 15 years and warm agglutinin AIHA on daily prednisone, who was sent to the emergency room by his primary care physician for evaluation of low hemoglobin. He also has symptoms of anemia, where he complained of increasing fatigue, weakness and shortness of breath on long distance walk. Otherwise, he denied palpitations, headache, and dizziness. He denied also mucocutaneous bleeding, hemoptysis, bloody vomiting, hematuria, hematochezia, and recent infections. He had the upper endoscopy performed in 2017, which showed chronically inflamed duodenal mucosa with extensive foveolar metaplasia and a small focus of gastric heterotopia. He has no history of colonoscopy for the past 10 years.

Initial vitals on admission were stable and physical examination was only notable for scleral icterus and conjunctival pallor. Digital rectal examination and fecal occult blood 
test were negative. His complete blood counts were significant for elevated white blood cell of $26.06 \times 10^{3} / \mu \mathrm{L}$ (predominantly neutrophilic), low hemoglobin $6.0 \mathrm{~g} / \mathrm{dL}$, low hematocrit $19.9 \%$, high mean corpuscular volume (MCV) of 133.1, high red cell distribution width (RDW) of 22.6 and low platelet $69 \times$ $10^{3} / \mu \mathrm{L}$. Initial blood chemistry showed elevated aspartate aminotransferase/alanine aminotransferase 152/83 U/L, elevated total bilirubin $2.2 \mathrm{mg} / \mathrm{dL}$, direct bilirubin was $0.47 \mathrm{mg} / \mathrm{dL}$. His direct antiglobulin test poly Coombs' test was positive, IgG was positive, C3 was negative, warm auto antibody was positive, PTT was low 20.4 s, PT/INR and fibrinogen were within normal limit. His reticulocyte count was significantly elevated $33.6 \%$, Haptoglobin was low $<7.75 \mathrm{mg} / \mathrm{dL}$, LDH was elevated 2,894 U/L. iron profile showed normal iron and iron saturation $(129 \mu \mathrm{g} / \mathrm{dL}, 70.1 \%)$, ferritin was elevated $523.5 \mathrm{ng} / \mathrm{mL}$, and total iron-binding capacity (TIBC) was low $184 \mu \mathrm{g} / \mathrm{dL}$. Septic workup was negative.

The patient was diagnosed with ES with coexistence of AIHA and ITP. Patient's platelets count prior to admission was in the range of $200-300 \times 10^{3} / \mu \mathrm{L}$. He was transfused with one unit of packed red blood cells (PRBC) and was given $125 \mathrm{mg}$ IV solumedrol in the emergency room. He was continued with solumedrol $80 \mathrm{mg}$ three times daily. Computed tomography (CT) chest/abdomen/pelvis showed mild splenomegaly, which was unchanged from before, there was no lymphadenopathy. The patient was responded to the treatment, his LDH and reticulocyte counts were trending down, and hemoglobin was improved to $7.7 \mathrm{~g} / \mathrm{dL}$. Patient was then given IVIG $1 \mathrm{~g} / \mathrm{kg}$ for two doses because his hemoglobin was still $<8 \mathrm{~g} / \mathrm{dL}$. Ultimately, he was discharged with $60 \mathrm{mg}$ prednisone daily for 2 weeks and follow-up in outpatient clinic. On 6 months' follow-up, his hemoglobin trended up to $12.4 \mathrm{~g} / \mathrm{dL}$ and platelets improved to $287 \times 10^{3} / \mu \mathrm{L}$.

\section{Discussion}

UC, a subgroup of inflammatory bowel disease with chronic inflammation mainly involving the large bowel and rectum, has been associated with numerous systemic autoimmune phenomena. Autoimmune hematologic disorders have been reported in patients with UC. AIHA has a lower incidence in patients with UC and has been reported only in $0.2-1.7 \%$ of patients [6]. Whereas, autoimmune induced thrombocytopenia is more common in this subgroup of patients [3]. Nevertheless, the presence of both acute autoimmune hemolysis and autoimmune induced thrombocytopenia in a patient with UC is extremely rare and to our knowledge, there was only one case report based on our literature search.

The coexistence of both AIHA and ITP is known as ES. It is first described by Robert Evans in 1951 after studying the relationship between AIHA and ITP. By definition, ES is an autoimmune condition that presents with two or more cytopenias, which includes warm AIHA and ITP with or without immune neutropenia. Patients with ES normally present with signs and symptoms related to anemia and thrombocytopenia which includes fatigue, pallor, shortness of breath, dizziness, enlarged spleen, easy bruising, petechiae and life-threatening bleeding. In patients older than 60 years, increased risk of ischemic complications has been reported like acute coronary and cerebrovascular syndromes [1].

ES in general is more common in children, but adults are also affected by ES. Mean age in adults at the time of diagnosis is 52. ES is more common in females with ratio of $3: 2$, likely related to the autoimmune diseases. ES is a sporadic condition and rarely inherited. Multiple pathways have been proposed for the pathophysiology of ES. These pathways summarize immune dysregulation, antibodies directed against erythrocytes, platelets and leukocytes. Some of the immune alterations associated with ES are deficiency of cytotoxic T-lymphocyteassociated protein 4 (CTLA-4)/CD152 and lipopolysaccharide-responsive and beige-like anchor protein ( $L R B A)$ gene, deficiency of tripeptidyl peptidase 2 and decreased CD4/CD8 ratio [2].

ES is classified as primary (idiopathic) and secondary (associated with other medical conditions). Although secondary ES responds to treatment better than primary ES [7], it has been shown that secondary ES has higher mortality rates with bleeding, infections and hematological cancers being the leading causes of death in these patients [8]. Secondary ES is very rare in patients with UC. Ucci et al was first to describe the co-occurrence of both ES and UC in a 37-year-old male. Ucci et al proposed that they might be two phenomena occurring in the same patient which leads to production of autoantibody directed to the red cells and platelets, leading to AIHA and ITP [5]. Whereas Jen et al described a case of vedolizumab-induced ES in a patient with UC [9]. Yates et al has shown that mononuclear cells from the colonic mucosa of UC patients can induce autoantibody formation which directed to the red cells leading to AIHA [10]. Whereas the same for ITP, bacterial glycopeptides have been hypothesized to play a major role in the formation of anti-platelet antibody [2]. These two distinct mechanisms may occur in the same patient, in certain circumstances, leading to the observed distinct phenomena of co-occurrence of both ES and UC in the same patient.

Our patient has a history of prostate cancer, and ES has been reported in an elderly patient with prostate cancer by Ureshino and Miyaraha [11]. Their patient initially developed AIHA and later developed ITP after trans-rectal ultrasonography guided prostate biopsy which resulted in transient elevation of prostate specific antigen (PSA), in which they hypothesized that the anti-antibodies directed against PSA may causing a destruction of platelets [11]. Moreover, PSA has been reported as a paraneoplastic antigen involved in AIHA and ITP $[12,13]$. Nonetheless, the prostate cancer was diagnosed 15 years ago and was in remission with no elevation of PSA.

Treatment in general involved the use of steroids (either prednisone or prednisolone) and IVIG. Patients will be reevaluated in 3 weeks after initial treatment. If patient responded to treatment, slow tapering of steroid is done over 6 months. Nonetheless, if partial response is noted, repeat dosing with full initial dose is normally performed. IVIG, on the other hand, can be used in patients who require high doses of steroids to attain remission, patients who develop adverse reaction to steroids and should be given to children in order to avoid the adverse toxicities related to steroids use [1]. 


\section{Conclusions}

Secondary ES is extremely rare in patients with UC. To our knowledge, this is the second case reporting the potential rare association between UC and ES. Recognizing this rare association is important as prompt treatment with IVIG and steroids will improve the prognosis and reduce the risk of complications.

\section{Acknowledgments}

None to declare.

\section{Financial Disclosure}

None to declare.

\section{Conflict of Interest}

All authors including Kok Hoe Chan, Su Lin Lim, Gowthami Are, Theodore DaCosta Jr and Hamid S Shaaban declare no competing conflict of interest.

\section{Informed Consent}

Patient has given the written informed consent to publish the case including publication of images.

\section{Author Contributions}

Kok Hoe Chan contributed to the conception ad design, acquisition, analysis and interpretation of data, as well as participated in drafting and revision of the manuscript. Su Lin Lim and Gowthami Are actively participated in analysis of data, drafting and revision of manuscript. Theodore DaCosta Jr and Hamid S Shaaban contributed to idea design, data analysis and critically revised the manuscript and as well as approved the final submission of manuscript.

\section{Data Availability}

The authors declare that data supporting the findings of this study are available within the article.

\section{References}

1. Michel M, Chanet V, Dechartres A, Morin AS, Piette JC, Cirasino L, Emilia G, et al. The spectrum of Evans syndrome in adults: new insight into the disease based on the analysis of 68 cases. Blood. 2009;114(15):3167-3172.

2. Wang W, Herrod H, Pui CH, Presbury G, Wilimas J. Immunoregulatory abnormalities in Evans syndrome. Am J Hematol. 1983;15(4):381-390.

3. Zlatanic J, Korelitz BI, Wisch N, Kim P, Ammirati M, Schwarz S, Gruenstein S, et al. Inflammatory bowel disease and immune thrombocytopenic purpura: is there a correlation? Am J Gastroenterol. 1997;92(12):2285-2288.

4. Norton A, Roberts I. Management of Evans syndrome. Br J Haematol. 2006;132(2):125-137.

5. Ucci G, Ferrando P, Valentini D, Zavallone L. A case of Evans' syndrome in a patient with ulcerative colitis. Dig Liver Dis. 2003;35(6):439-441.

6. Giannadaki E, Potamianos S, Roussomoustakaki M, Kyriakou D, Fragkiadakis N, Manousos ON. Autoimmune hemolytic anemia and positive Coombs test associated with ulcerative colitis. Am J Gastroenterol. 1997;92(10):1872-1874.

7. Costallat GL, Appenzeller S, Costallat LT. Evans syndrome and systemic lupus erythematosus: clinical presentation and outcome. Joint Bone Spine. 2012;79(4):362364.

8. Hansen DL, Moller S, Andersen K, Gaist D, Frederiksen H. Evans syndrome in adults - incidence, prevalence, and survival in a nationwide cohort. Am J Hematol. 2019;94(10):1081-1090.

9. Jen H, Morganstern B, D'Souza L. A Case of Evan's Syndrome Induced by Vedolizumab. ACG Case Rep J. 2019;6(1):e00002.

10. Yates P, Macht LM, Williams NA, Elson CJ. Red cell autoantibody production by colonic mononuclear cells from a patient with ulcerative colitis and autoimmune haemolytic anaemia. Br J Haematol. 1992;82(4):753-756.

11. Ureshino H, Miyahara M. Unusual co-occurrence of Evans syndrome and pure red cell aplasia in elderly patient with prostate cancer. Ann Hematol. 2016;95(10):17431744.

12. Puthenparambil J, Lechner K, Kornek G. Autoimmune hemolytic anemia as a paraneoplastic phenomenon in solid tumors: A critical analysis of 52 cases reported in the literature. Wien Klin Wochenschr. 2010;122(7-8):229236.

13. Pelosof LC, Gerber DE. Paraneoplastic syndromes: an approach to diagnosis and treatment. Mayo Clin Proc. 2010;85(9):838-854. 